ASP Conference Series, Vol. 0, 2000

.

\title{
Simulations of Merging Clusters of Galaxies
}

\author{
Sabine Schindler \\ Astrophysics Research Institute, Liverpool John Moores University, \\ Twelve Quays House, Birkenhead CH41 1LD, U.K.
}

\begin{abstract}
Numerical simulations of cluster mergers reveal many characteristics of the merging process: shock structure and strength, observational signatures of the dynamical state, effects on the mass determination, turbulence and the evolution of the X-ray luminosity and the magnetic field. In this article I review the results obtained from various simulations over the last years.
\end{abstract}

\section{Introduction}

Irregular cluster morphologies in many X-ray images of cluster as well as indications from optical observations imply that many clusters are not relaxed. Hence merging is a common phenomenon in clusters of galaxies. Such mergers of subclusters are very energetic events, which affect clusters strongly, e.g. shocks emerge which are important because they are the major heating source for the intra-cluster gas and moreover they can accelerate particles.

While observations provide only snapshots of the different evolutionary stages of mergers, simulations are the only way to follow the evolution. The models reveal where shocks emerge, where they move and how strong they are. With the help of simulated X-ray images at different stages of a merger a connection between X-ray morphology and the dynamical state can be established and the effects on mass determination can be investigated. Magnetohydrodynamic simulations show how the magnetic field is enhanced during mergers and how the overall structure of the magnetic field is changed due to a merger.

\section{Simulation Methods}

In order to perform realistic simulations three-dimensional calculations are required and the different cluster components must be taken into account. It is necessary to follow the evolution of the dark matter as well as the intra-cluster gas. Dark matter (and galaxies) can be regarded as collisionless particles and can therefore be modeled by N-body simulations. The gas is best simulated by hydrodynamic calculations. For these hydrodynamic calculations two different methods have mainly been used: (1) Smoothed Particle Hydrodynamics (Lagrangian approach, e.g. Evrard 1990; Dolag et al. 1999; Takizawa 1999; Takizawa \& Naito 2000) in which the gas is treated as particles and (2) grid codes (Eulerian approach, e.g. Schindler \& Müller 1993; Bryan et al. 1994; Roettiger et al. 1996, 1997, 1998, 1999a,b; Ricker 1998; Quilis et al. 1998) 
in which the simulation volume is divided into cells. Fortunately, the choice of simulation method seems to be irrelevant. Calculations with both methods yield very similar results.

\section{Shocks}

Mergers produce shocks in the intra-cluster gas. These shocks are of particular interest for particle acceleration models. The strongest shocks emerge after the collision of subclusters, when these shock propagate outwards along the original collision axis (Schindler \& Müller 1993; Roettiger et al. 1999a). However, even these shocks are relatively mild shocks with a maximum Mach number of about 3 . When a dense subcluster falls into a cluster a shock emerges already before the core passage: a bow shock is visible in front of the infalling subcluster (Roettiger et al. 1997). In general, the shock structure is found to be more filamentary at early epochs and quasi-spherical at low redshifts (Quilis et al. 1998).

Observationally, the shocks are best visible in temperature maps, because they show up as steep temperature gradients. For such maps spatially resolved $\mathrm{X}$-ray spectroscopy is necessary which can be performed now with high accuracy with the new X-ray observatories XMM and Chandra.

\section{Other Effects of Mergers}

Apart from shocks, mergers have many other interesting effects. For example, the X-ray luminosity increases during the collision of two subclusters (Schindler \& Müller 1993). The reason is that the gas is compressed, i.e. the gas density is increased, and as the X-ray emission is proportional to the square of the density we see enhanced X-ray emission during the passage. During the core passage and at each rebounce an increase in the magnetic field is visible (Dolag et al. 1999, 2000 (in prep.); Roettiger et al. 1999b; see also Section 6). Mergers also cause a lot of turbulence. Off-centre collisions produce in addition angular momentum (Ricker 1998; Roettiger et al. 1998). The shocks heat primarily the ions as has been shown in simulations which treat ions and electrons separately (Chièze et al. 1998; Takizawa 1999).

Observationally mergers cannot only be identified by multiple X-ray maxima, but also by isophote twisting with centroid shift and elongations: the dark matter component is always elongated along the collision axis, while the gas is first elongated along the collision axis but during the core passage it is pushed out perpendicular to the collision axis, so that later an elongation perpendicular to the collision axis can be seen (Schindler \& Müller 1993). Also offsets between the collisionless component and the gas have been found (Roettiger et al. 1997).

\section{Mass Determination in Merging Clusters}

Mass determination in clusters with the X-ray method can be affected strongly during mergers (Evrard et al. 1996; Roettiger et al. 1996; Schindler 1996). The reason is that during the merging process there can be quite strong deviations from two assumptions necessary for the mass determination - hydrostatic 
equilibrium and spherical symmetry. For example, at the positions of shocks the gas is not in hydrostatic equilibrium. Shocks cause gradients - both in the temperature and in the density - and can cause therefore an overestimation of the mass. Locally, this can lead to a mass estimate up to two times the true mass. Substructure on the other hand tends to flatten the azimuthally averaged profile and hence leads to an underestimation of the mass, in extreme cases to deviations of $50 \%$ of the true mass.

In some cases, these deviations can be corrected for, e.g. in clusters in which substructures are well distinguishable, the disturbed part can be excluded from the mass analysis and a good mass estimate can be obtained. But in general, mass determinations in non-relaxed clusters should be done very cautiously.

\section{Simulations of Mergers with Magnetic Fields}

Radio halos require the existence of magnetic fields in clusters on scales of a few Mpc. Therefore magnetohydrodynamic calculations have been performed (Dolag et al. 1999; Roettiger et al. 1999b). It has been found that the initial field distribution is irrelevant for the final structure of the magnetic field. The structure is dominated only by the cluster collapse. Faraday rotation measurements can be reproduced by the simulations for magnetic fields of the order of $\mu \mathrm{G}$. Most important for the amplification of the magnetic field are shear flows, while the compression of the gas is of minor importance. Mergers change the local magnetic field strength as well as the structure of the cluster-wide field. At early stages of the merger, filamentary structures prevail, which break down later and leave a stochastically ordered magnetic field.

\section{References}

Bryan, G.L. et al. 1994, ApJ, 437, L5

Chièze, J.-P., Alimi J.-M., Teyssier, R. 1998, ApJ, 495, 630

Dolag, K., Bartelmann, M., Lesch, H. 1999, A\&A, 348, 351

Evrard, A.E. 1990, A\&A, 363, 349

Evrard, A.E., Metzler, C.A., Navarro, J.N. 1996, ApJ, 469, 494

Quilis, V., Ibáñez, J.M., Sáez, D. 1998, ApJ, 502, 518

Ricker, P.M. 1998, ApJ, 496, 670

Roettiger, K., Burns, J.O., Loken, C. 1996, ApJ, 473, 651

Roettiger, K., Burns, J.O., Stone J.M. 1999a, ApJ, 518, 603

Roettiger, K., Loken, C., Burns, J.O. 1997, ApJS, 109, 307

Roettiger, K., Stone J.M., Burns, J.O. 1999b, ApJ 518, 594

Roettiger, K., Stone J.M., Mushotzky, R.F., 1998, ApJ, 493, 62

Schindler, S. 1996, A\&A, 305, 756

Schindler, S., Müller, E. 1993, A\&A, 272, 137

Takizawa, M. 1999, ApJ, 529, 514

Takizawa, M., Naito, T., 2000, ApJ, 535, 586 